\title{
Large-Eddy Simulation of Turbulence in Cardiovascular Flows
}

\author{
F. Nicoud, C. Chnafa, J. Siguenza, V. Zmijanovic and S. Mendez
}

\begin{abstract}
A 4th-order accurate, low dissipative flow solver is used to perform Large-Eddy Simulations of three typical hemodynamic situations: the flow through the idealized medical device proposed by the American Food and Drug Administration; the intracardiac flow within an actual human left heart whose morphology and deformations are deduced from medical imaging; the flow downstream of an artificial aortic valve which arises from the blood-leaflets interaction problem. In all the cases, the $\sigma$ subgrid scale model designed to handle wall-bounded transitional flows is successfully used and the numerical simulations compare favourably with the experimental data available. These results illustrate the potential of the LargeEddy Simulation methodology to properly handle blood flows. They also support the idea that turbulence, even if not fully developed, may be present in cardiovascular flows, including under non pathological conditions.
\end{abstract}

\section{Introduction}

Turbulence is a natural phenomenon in fluid mechanics, which gives rise to wide spectrum, chaotic fluctuations in the velocity/pressure fields, even in the absence of external forcing. This phenomenon arises when the inertia forces are significantly larger than the viscous effects, viz. when the Reynolds number $R_{e}$ exceeds a few thousands in steady flow, most probably less for pulsatile flows [1]. This number usually reaches a few hundreds in medium-sized arteries and a few thousands in the largest vessels, in the heart itself or within biomedical systems such as total artificial hearts or ventricular assist devices. Thus, one may expect turbulence to be present in

F. Nicoud $(\bowtie) \cdot$ J. Siguenza $\cdot$ V. Zmijanovic $\cdot$ S. Mendez

Institut Montpelliérain Alexander Grothendieck, CNRS, Univ. Montpellier,

Montpellier, France

e-mail: franck.nicoud@umontpellier.fr

C. Chnafa

Biomedical Simulation Laboratory, Department of Mechanical \& Industrial Engineering,

University of Toronto, Toronto, Canada

e-mail: cchnafa@mie.utoronto.ca 
the cardiovascular system, at least in some favourable regions in space (e.g. close to stenoses, valves, bifurcations) and during specific periods of the cardiac cycle (when the flow decelerates). Still, until recently turbulence was virtually not studied, at least non-invasively, in the cardiovascular biomechanics community, notably because in vivo $3 \mathrm{D}$ and unsteady measurements of the blood velocity are very challenging. Recent progresses in both computational fluid dynamics (CFD) and medical imaging techniques give rise to a renewed interest for the turbulent properties of blood flows. Unstable and/or turbulent flows have been observed in numerical simulations in abdominal aortic aneurysms [2], carotid siphons [3], cerebral aneurysms [4] as well as experimentally in an idealized left ventricle [5]. Thanks to an MRI method based on the intravoxel velocity standard deviation, it is now possible to estimate the turbulent kinetic energy in vivo. Significant levels of kinetic energy were found not only in pathological left atria [6] but also in normal left ventricles [7], supporting the idea that turbulence can also occur in healthy human subjects.

From a numerical point of view, representing turbulence is a challenge due to the wide variety of both temporal and spatial scales that are present at the same time. In the ideal case of a decaying isotropic turbulence, one may show that the largestto-smallest length scales ratio behaves like $R_{e}^{3 / 4}$ [8]; as a consequence, the number of grid points necessary to properly represent a turbulent flow, including the whole range of fluctuations, increases as fast as $R_{e}^{9 / 4}$. This approach where turbulence is accounted for by just and only solving all the scales produced by the Navier-Stokes equations is called Direct Numerical Simulation and remains most of the times out of reach of the current computing power except in simple cases with moderate Reynolds number. The large variety of scales inherent to any turbulent flow motivated the development of turbulent models in order to complement the Reynolds-Averaged Navier-Stokes (RANS) equations which allow to compute the averaged flow quantities without explicitly representing the fluctuations. Despite decades of very active research and some successes in fully developped turbulent flows, there are still many controversies about the proper way to develop 'good' turbulent models [9] and the idea of a universal formulation able to properly deal with any turbulent flows is widely considered as hopeless. On top of not being predictive enough, RANS models are better suited for fully turbulent flows at very high Reynolds numbers than transitional flows. The ability of Large-Eddy Simulation (LES) to better predict a flow without prior knowledge, including regarding its laminar/turbulent nature, is one of the reasons why this technique is now being used in many applications. It is a method of choice for unsteady 3D flows at moderate Reynolds numbers, especially when transition from laminar to turbulence plays a key role. In this paper, we illustrate how LES can be used to represent three configurations typical of cardiovascular applications:

- an idealized medical device where a stationary flow goes from laminar to turbulent in a simple geometry [10],

- a realistic human left heart whose geometry and cyclic deformations are deduced from medical imaging and where CFD/LES in a time varying domain is used to generate the associated blood flow [11], 
- the flow past an artificial aortic valve where the Fluid-Structure Interaction problem is solved to predict the leaflets dynamics and associated turbulent generation.

Some numerical and physical issues specific to each of these configurations will be discussed in Sects. 3, 4 and 5 respectively, together with comparison with experimental data when available. Some details about the numerics and the subgrid scale modelling strategy are first given in Sect. 2.

\section{LES Requirements}

In LES, the modelling effort focuses on the smallest length scales of fluctuations which are presumably less dependent on the geometry of the flow domain and thus more universal. The largest scales (which can be as small as twice the typical cell size, at least in theory) are explicitly resolved as solutions of the low-pass filtered Navier-Stokes equations [12]. Thus, the finer the mesh, the larger the range of resolved scales and the smallest the contribution of the subgrid scale model. In other words, the modelling effort in less intense in LES compared to RANS and this is all the more true that the grid is refined.

The price to pay is that the dynamics of the largest scales, as well as the related macroscopic mixing and turbulent stresses, must be properly represented by the numerics. As a consequence the numerical errors associated to both the spatial and temporal discretizations must be kept as small as possible. Notably, dissipation-free schemes must be preferred so that the (relative) intensity of the different resolved scales is not spoiled. An important feature of LES is that the scheme should be as accurate as possible for all the scales represented on the mesh, the largest as well as the smallest. This means that the order of accuracy of a numerical method is not a good measure of its capability to perform well in the LES; the effective wave number [13] and kinetic energy conservation [14] concepts are better suited to characterize a numerical scheme dedicated to LES. For example, better results may be obtained with a second order centered (dissipation-free at all scales) scheme than with a higher-order upwind biased (dissipative) scheme [15, 16]. As a consequence, a RANS solver, usually based on stabilized scheme coupled to implicit time marching methods designed to speed up the convergence towards a steady solution cannot be easily adapted to LES. In the present study, the in-house flow solver YALES2BIO (http://www.math.univ-montp2.fr/ yales2bio) was used to solve the filtered flow equations. Dedicated to the computations of blood flows, this general purpose solver relies on a 4th-order accurate centred (dissipation-free) finite-volume formulation where the projection method [17] is used to meet the divergence-free condition. The time-stepping is also 4th-order accurate, based on the dissipationfree explicit low-storage Runge-Kutta scheme [18]. At the end, the algorithm is non dissipative and one relies on the physical dissipation, either laminar or issued by the subgrid scale model, to ensure numerical stability. This is made possible because the Reynolds number is low to moderate in biomedical applications so that numer- 
ical stabilization is not necessary; this also requires to use high quality 3D meshes. Finally, YALES2BIO inherits its parallel efficiency from the YALES2 package developed by V. Moureau and co-workers [19, 20].

From the modelling point of view, the subgrid scale stress tensor $\tau_{S G S}$ which results from the filtering operation applied to the Navier-Stokes equations is most of the time modelled thanks to the eddy-viscosity assumption (introduced in the RANS concept in the 70s). In the case of an incompressible fluid, this reduces to

$$
\tau_{S G S}=2 \rho v_{S G S} \mathbf{S}
$$

where $\rho$ is the fluid density, $\mathbf{S}$ is the strain rate tensor of the resolved scales and $v_{S G S}$ is the so-called subgrid scale viscosity. Several models were proposed over the years for this quantity and most of them share the following form:

$$
v_{S G S}=(C \Delta)^{2} \mathscr{D}(\mathbf{u})
$$

where $C$ is the model constant which is usually tuned (either theoretically or numerically) so that the model produces the proper amount of dissipation in the simple case of decaying isotropic turbulence. The length scale $\Delta$ denotes the typical size of the local cell of the mesh used to solve the filtered Navier-Stokes equations and $\mathscr{D}$ is a differential operator which defines the model and operates on the resolved velocity field $\mathbf{u}$. The $\boldsymbol{\sigma}$-model [21] is used in this study, meaning that:

$$
\mathscr{D}(\mathbf{u})=\frac{\sigma_{3}\left(\sigma_{1}-\sigma_{2}\right)\left(\sigma_{2}-\sigma_{3}\right)}{\sigma_{1}^{2}}
$$

In this expression, $\sigma_{1} \geq \sigma_{2} \geq \sigma_{3} \geq 0$ are the three singular values of the local velocity gradient tensor and can be efficiently computed [21]; the model constant is $C=1.35$. This model was selected because it meets several useful properties relevant in terms of SGS modelling, although not shared by the other SGS models. On top of vanishing for a variety of canonical laminar flows for which no SGS viscosity is expected, it has the proper cubic behavior in near-wall regions. In [21], the model was implemented in different numerical solvers and validated in academic cases (homogeneous isotropic turbulence, turbulent channel); its effectiveness in a more demanding configuration was also demonstrated recently by considering the impingement of an unsteady jet with a rigid wall [22]. In this particular case which is relevant to intra-cardiac hemodynamics, the comparison with experimental data shows that the $\boldsymbol{\sigma}$-model outperforms the well-known Dynamic Smagorinsky model [23].

In the next three sections, results from the $\sigma$-model as implemented in the YALES2BIO solver are discussed to illustrate how LES can be used to analyse flows relevant to cardiovascular applications. 


\section{The FDA Medical Device Test Case}

The United States Food and Drug Administration (FDA) medical device benchmark was introduced at the beginning of the current decade as an answer to the ubiquitous presence of various numerical methods in biomedicine. The purpose of the proposed benchmark program was to validate the CFD codes on idealized medical device examples. The first FDA test model [10, 24] aims at challenging CFD codes on a case whith transition to turbulence, which is frequently encountered in biomedical devices. Accurately predicting turbulence is necessary to properly assess shear stresses, themselves relevant to blood damage prediction in ventricular assist devices (VAD) $[25,26]$, but also in blood flows through stents and pumps, to cite a few. Mispredicting the turbulent nature of blood flows heavily impacts the results and may cause critical faulty conclusions.

The FDA medical device model, as illustrated in Fig. 1, consists of a fixed axisymmetric geometry with a long inlet section, a convergent nozzle, a 10 diameters long throat section and a sudden expansion at the downstream end. This geometry submits the flowing fluid to several events as acceleration through the convergent, pipe type of flow in the throat where maximal Reynolds number $\left(\operatorname{Re}_{t h}\right)$ is reached and jet formation into the sudden expansion section characterized by deceleration, mixing shear layer generation and possible turbulence transition. Five flow regimes were considered, which correspond to fully laminar case $\left(\operatorname{Re}_{t h}=500\right)$, transient case $\left(\operatorname{Re}_{t h}=2000\right)$, laminar inlet—-transition to turbulence in sudden expansion $\left(\mathrm{Re}_{t h}=3500\right)$, laminar inlet-turbulent case $\left(\operatorname{Re}_{t h}=5000\right)$ and fully turbulent case $\left(\operatorname{Re}_{t h}=6500\right)$.

The reported computational investigations on this FDA benchmark can be divided into two major groups: blinded studies that were conducted without foreknowledge of the experimental results and the studies conducted after the publication of first benchmark results. While none of the first 28 CFD research groups which used various RANS methods managed to fully reproduce the FDA experimental results [24], almost all succeeding CFD studies [27-30] reported good agreement with the FDA experiments. This raises a number of questions regarding both the numerical

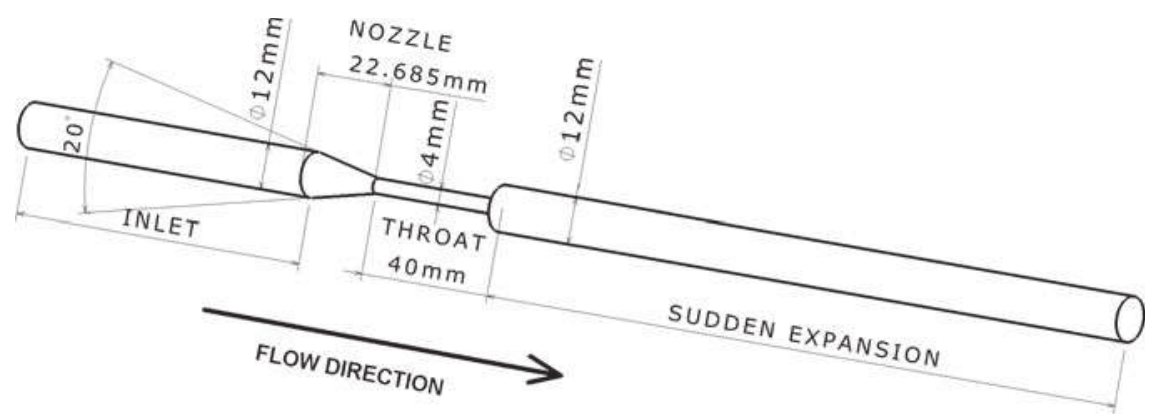

Fig. 1 Geometry specifications of the first FDA ideal medical device 
methods for turbulence transitional blood flows and the overall methodology to represent the FDA blood flow case. While the RANS codes [24, 31] in this case clearly lag behind the LES due to their inability to accurately predict the laminar to turbulent transition, the absence of analysis of the sensitivity to numerical parameters in the reported DNS and LES results [27, 28, 30] questions the actual predictive character of these simulations. Indeed, the FDA case proves to be highly sensitive to any physical or numerical specificities as illustrated in the remaining of this section.

\subsection{Simulations with Perturbation-Free Inlet}

Investigation of the effects of numerics on the results was conducted by considering three grid levels as well as a variety of CFL numbers (dimensionless time step). In order not to presume the resulting flow field in any way, unstructured finite volume grids were designed with almost no-local refinement in the domain with only slight stretching towards the upstream and downstream ends. The three grids contain respectively 5 million tetrahedral elements with $h=0.34 \mathrm{~mm}$ average cell height (Coarse), 15 million with $h=0.2 \mathrm{~mm}$ (Medium) and 50 million with $h=0.14 \mathrm{~mm}$ (Fine). Further details on the numerics and grids are available in [32].

Snapshots of instantaneous velocity fields obtained with the medium grid are displayed in Fig. 2 for the transitional $\left(\operatorname{Re}_{t h}=3500\right)$ case. Except for the CFL number, the same physical and numerical conditions were used in both cases. This figure illustrates that the location of the transition to turbulence is very sensitive to the numerical details, as confirmed by the profiles displayed in Fig. 3. The latter also shows that the

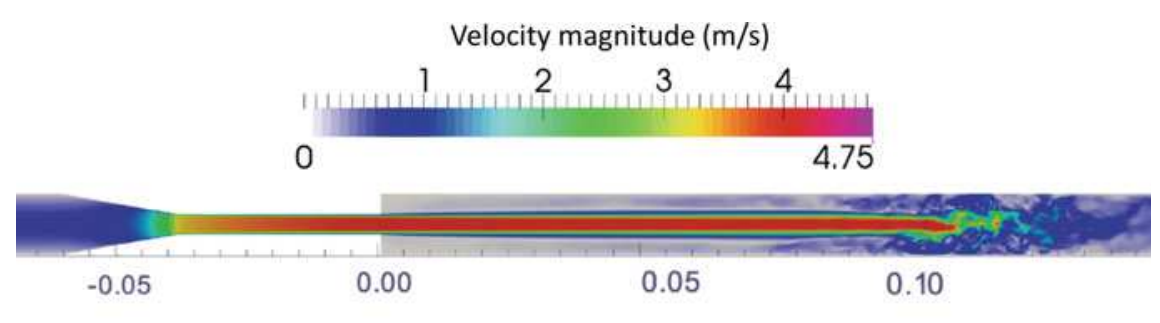

(a) $\mathrm{CFL}=0.6$

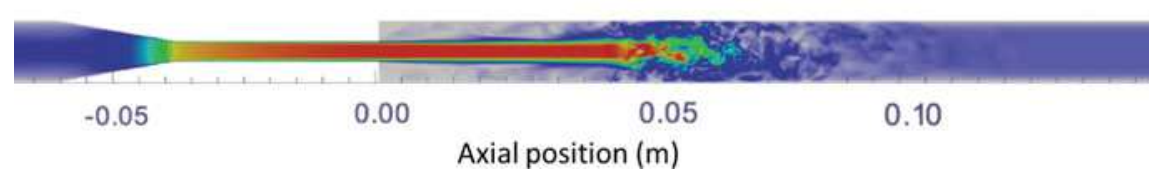

(b) $\mathrm{CFL}=0.1$

Fig. 2 Instantaneous velocity fields (medium grid) for two different values of the CFL numbers (top $\mathrm{CFL}=0.6$; bottom $\mathrm{CFL}=0.1)-\mathrm{Re}_{\text {th }}=3500$ 
Large-Eddy Simulation of Turbulence in Cardiovascular Flows

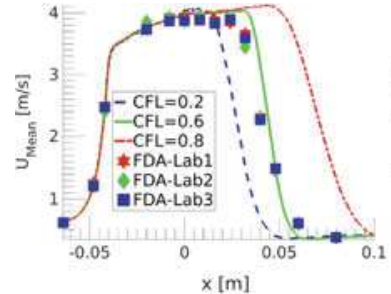

(a) Velocity - Coarse grid

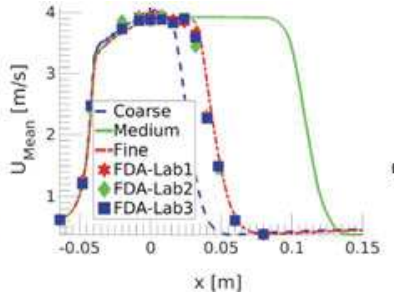

(b) Velocity $-\mathrm{CFL}=0.6$

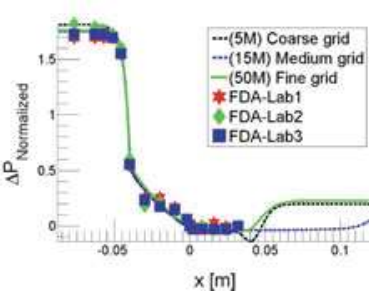

(c) Pressure - CFL $=0.6$

Fig. 3 Longitudinal evolutions of the streamwise velocity (left and middle plots) and wall-pressure (relative to the pressure at $\mathrm{x}=0$ and normalized by the dynamic pressure at the throat - right $\mathrm{plot}$ ) for a variety of temporal and spatial resolutions- $-\mathrm{Re}_{t h}=3500$

upstream part of the flow ( $x<0$, the sudden expansion starts at $\mathrm{x}=0$ ) is robust to the details of the simulation. However, both the streamwise mean velocity and the static pressure strongly depend on the numerics in the downstream region. This means that the axisymmetric configuration considered, when fed with an ideal Poiseuille velocity profile without perturbation, is very sensitive to any physical (boundary conditions) or numerical (mesh stretching, schemes, time step) modification. This makes hopeless any effort towards a predictive simulation of this configuration. A cure to this issue is considered in the next section.

\subsection{Small Perturbations at the Inlet}

When dealing with transitional flows, the perturbations injected at the inlet of the computational domain are of prime importance as already discussed in the literature [33, 34]. In the particular case of the FDA nozzle, the absence of such inlet perturbations leads to an extreme sensitivity of the results which makes the simulations unreliable. Note that this may also lead to simulations which may compare well with the experimental data set but which are nonetheless inadequate since not robust (e.g. the case $\mathrm{CFL}=0.6$ and coarse grid in Fig. 3a agrees well with the measurements but should not be trusted since the results depend on the time step).

As shown in Fig. 4, even the smallest amount of perturbations injected at the inlet (thereafter denoted turbulence injection, TI) greatly improves the situation in the sense that the results are now robust to any tested numerical condition. More importantly, the numerical results are also systematically in good agreement with the experimental data, suggesting that the simulations outcomes are both robust and accurate. Profiles of the root-mean-square (RMS) velocity, Fig. 5a, show that the amount of flow perturbations at inlet is significantly increased by TI, as expected. The small amplitude perturbations are quickly dissipated downstream, being decreased by more than 2 orders of magnitude as shown in Fig. 5b. Figure $5 \mathrm{c}$ and $\mathrm{d}$ show that the flow in the throat is characterized by an increase of the near wall RMS. Just after the 


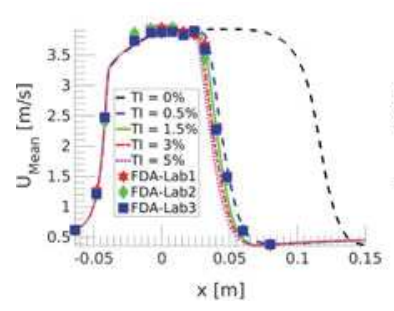

(a) $\mathrm{CFL}=0.6$ : TI range

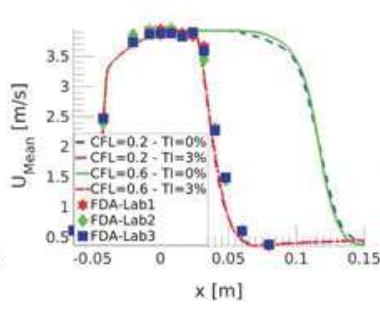

(b) CFL vs TI levels

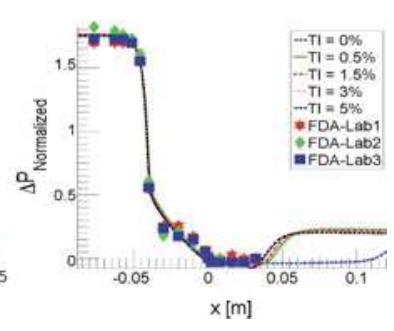

(c) Pressure, $\mathrm{CFL}=0.6$ : $\mathrm{TI}$ range

Fig. 4 Mean axial velocity and pressure profiles with small TI at inlet (Medium grid, $\mathrm{Re}_{t h}=3500$ )

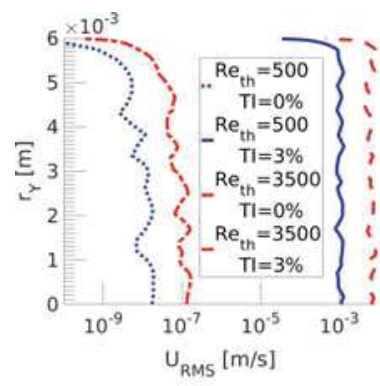

(a) $\mathrm{x}=-0.15 \mathrm{~m}$ (Inlet)

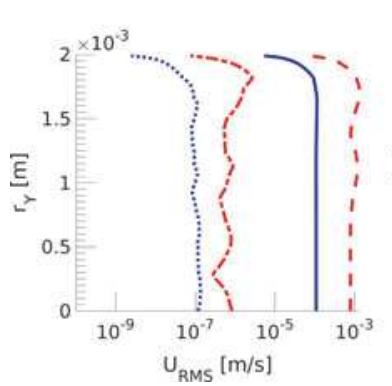

(d) $\mathrm{x}=-0.008$ (throat)

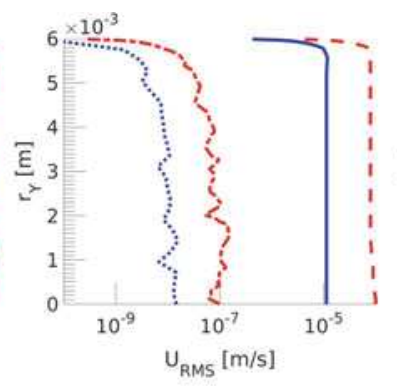

(b) $\mathrm{x}=-0.088 \mathrm{~m}$ (Inlet nozzle)

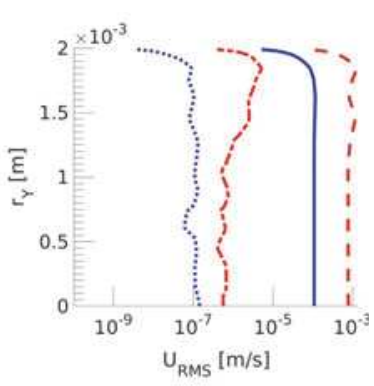

(c) $\mathrm{x}=-0.034$ (throat)

Fig. 5 Radial profiles of the root-mean-square (RMS) velocity for the laminar $\left(\operatorname{Re}_{t h}=500\right)$ and transitional $\left(\mathrm{Re}_{t h}=3500\right)$ regimes at a variety of axial positions with and without TI (Medium grid)

sudden-expansion, Fig. 5e and f, both RMS profiles obtained with TI are approaching the original no TI cases before merging into laminar flow profile $\left(\operatorname{Re}_{t h}=500\right)$ or advancing into turbulence breakdown $\left(\mathrm{Re}_{t h}=3500\right)$.

Figure 6 shows that TI greatly improves the LES prediction as well as its robustness for laminar, mildly turbulent cases and even in the most demanding, transitional case $\left(\operatorname{Re}_{t h}=2000\right)$. The injected perturbations dissipate without inflicting transition in the laminar case, while in the transitional and turbulent cases they develop and contribute to more accurate and robust results. 


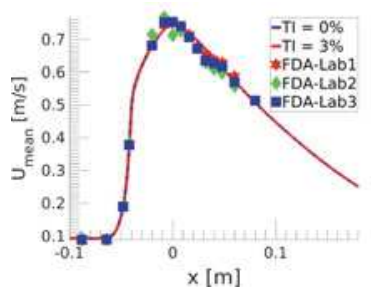

(a) $\mathrm{Re}_{t h}=500$

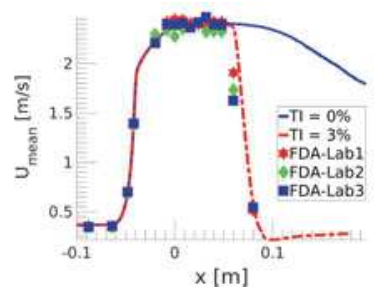

(b) $\mathrm{Re}_{t h}=2000$

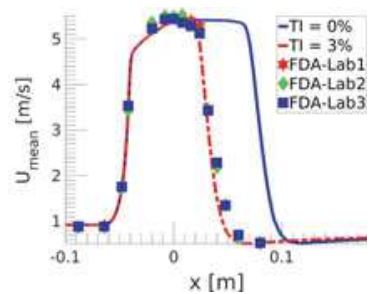

(c) $\mathrm{Re}_{t h}=5000$

Fig. 6 Axial velocity profiles for the laminar, transitional and mildly turbulent cases with and without TI (Medium grid)

The study thus reveals that the upstream injection of small-scale perturbations into transition sensitive flows is an indispensable tool for any biomedical case where accurate prediction of turbulence is critical.

\section{Intracardiac Turbulence}

The hemodynamics of the left heart (LH) conveys information regarding the heart function $[35,36]$. Observation of LH flow may thus reflect the presence of an existing pathology. In addition, there is substantial evidence that the LH hemodynamics can be responsible for the initiation of ventricular remodelling through mechanosensitive feedbacks modulating cardiomyocytes architecture $[37,38]$ and thus cardiac function [39]. Therefore, accurate assessment of the intracardiac flow is of paramount importance to get further comprehension of the role played by the hemodynamics in normal and abnormal LH. Used with caution, image-based computational fluid dynamics (CFD) can retrieve all the scales of the instantaneous flow, hence being able to capture disturbed flows. However, pioneering CFD studies focused only on the large-scale features of the ventricular flow i.e. the jets and the large recirculating cell. Apart from a few studies that mentioned the potential presence of flow instabilities [11, 40-42], little focus has been given to the disturbed nature of the LV flow, despite its potential importance.

\subsection{Method}

Large-eddy simulations were preformed to compute 35 cardiac cycles using the inhouse YALES2BIO solver in a patient-specific left heart model obtained from CMR images. The set of images used to define the time evolution of the model over the cadiac cycle consists in $203 \mathrm{D}$ images of spatial resolution $5.0 \times 1.1 \times 1.1 \mathrm{~mm}^{3}$ $(21 \times 256 \times 256$ voxels $)$. The subject was 26 years old and his cardiac cycles lasted 

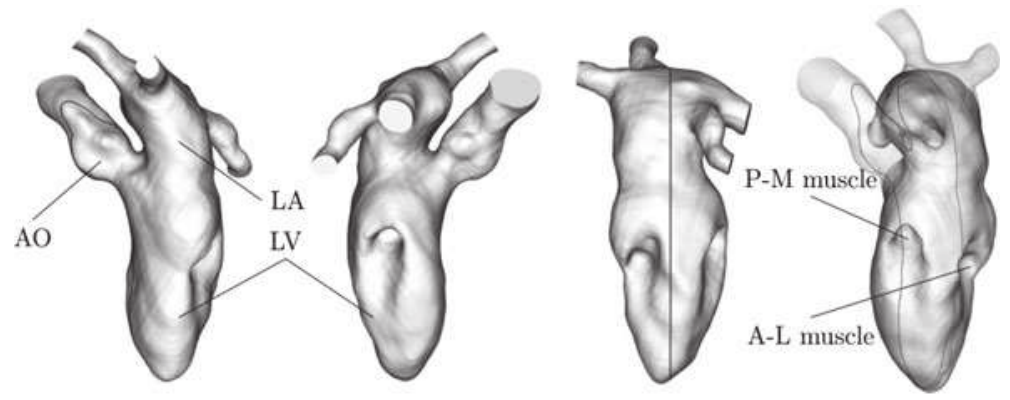

Fig. 7 Human left heart extracted from CMR images. The same domain is shown from four different points of view. The inlets and outlet flow extensions are visible in the left figure. The left ventricle (LV), left atrium (LA), Aorta (AO), Antero Lateral (A-L) and Postero Medial (P-M) papillary muscles are indicated. A black line passing through the LH indicates the position of the slice which will be used to display the results in Figs. 8 and 10

on average $\mathrm{T}=750 \mathrm{~ms}$. As shown in Fig. 7, the model includes the valves, atrium, ventricle, papillary muscles and ascending aorta. The deformations of the heart all along the heart cycle match the CMR exam thanks to an image registration technique. The technical procedure can be found in $[11,43]$. The flow domain within the heart is discretized using ten million tetrahedral elements. The average edge length is close to $0.55 \mathrm{~mm}$ during diastole. The time step, based on a Courant-Friedrichs-Lewy stability number of 0.9 , varies from $0.2 \mathrm{~ms}$ during the beginning of diastole to $0.5 \mathrm{~ms}$ during diastasis.

\subsection{Results}

The LV large-scale flow features i.e. the blood ejection, the two vortex rings for the $\mathrm{E}$ and A waves and the recirculating cell, are well retrieved (Fig. 8) in accordance with the numerous observations performed in vivo [42, 44], in silico [40, 45] and in vitro [46, 47]. However, our computation reveals also large velocity fluctuations, which are usually not reported in silico but which are in line with the results of previous experimental work that used both simplified ventricle geometries and inflow boundary condition $[5,47]$. In order to quantify and study the regional distribution of these fluctuations, we computed them as the difference between the instantaneous velocity components $u_{i}(i=1,2,3)$ and the phase-averaged velocity components $U_{i}$. The fluctuating part of the fluid velocity is then:

$$
u_{i}^{\prime}=u_{i}-U_{i}=u_{i}-\left\langle u_{i}\right\rangle
$$

while the corresponding fluctuating kinetic energy (FKE) per unit volume is: 


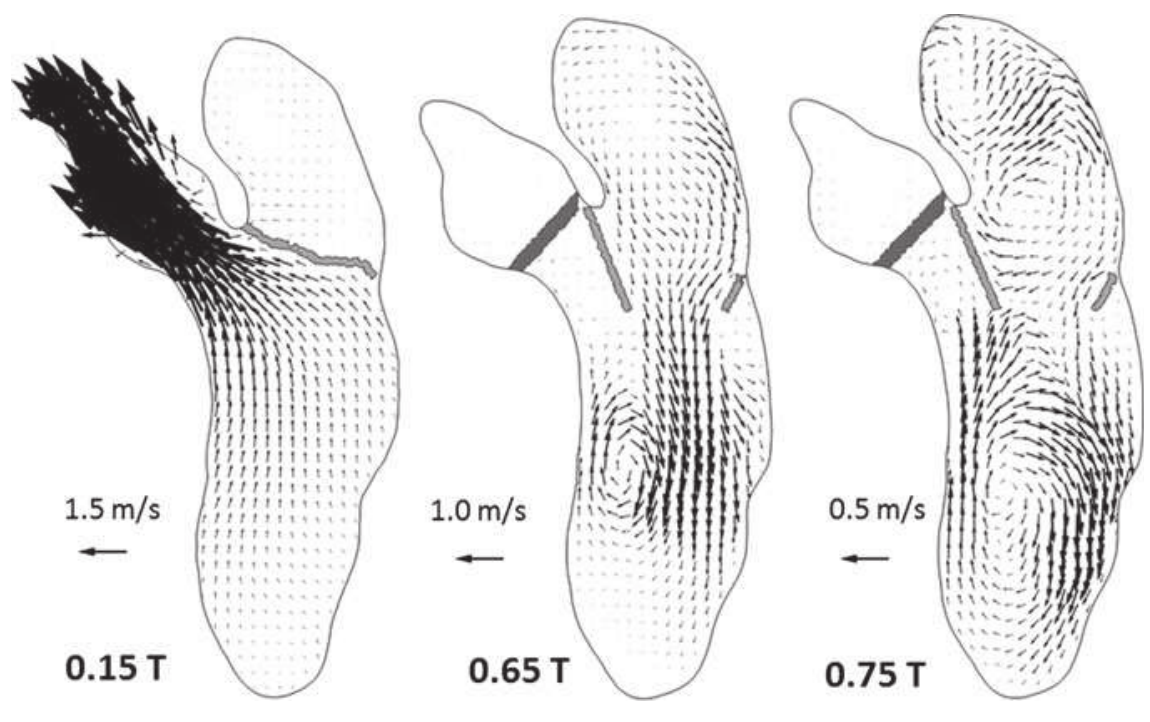

Fig. 8 Phase-averaged velocity field over a cutting plane through the LH (see Fig. 7 for the plane position). The mitral and the aortic valves are depicted in grey. The figure shows the classically reported structure of the heart cycle: the strong blood ejection at systole $(0.15 \mathrm{~T})$, the $\mathrm{E}$ waveinduced vortex ring at diastole $(0.65 \mathrm{~T})$ and the recirculating cell during diastasis $(0.75 \mathrm{~T})$

$$
F K E=\frac{\rho}{2}\left\langle u_{i}^{\prime} u_{i}^{\prime}\right\rangle
$$

where \langle\rangle denotes phase-averaged values, $\rho$ is the fluid density (here $1040 \mathrm{~kg} / \mathrm{m}^{3}$ ) and the implicit summation rule over repeated indices is used. Figure 9 displays the FKE in the left heart during systole (left figure) and diastasis (right figure). Note that non negligible levels of FKE are visible at these instants. Detailed results of this simulation and its biomedical consequences can be found in [41].

\subsection{Role of SGS Model}

As a common practice when using properly resolved LES, all quantities are computed from the resolved velocity field in this study [48, 49]. We note that during our computations the subgrid scale viscosity remains low in the LV during the whole the heart cycle, showing that the subgrid scale model dissipates a moderate amount of energy. Figure 10 shows the ratio between the subgrid scale viscosity and the fluid viscosity. As expected, since the flow is laminar, the subgrid scale viscosity is virtually zero during the systole in the LV and has moderate values, but non-null, during the most turbulent part of the cycle. Thus, the $\sigma$-model [21] mainly introduces dissipation in regions where there are significant turbulent fluctuations and 
F. Nicoud et al.
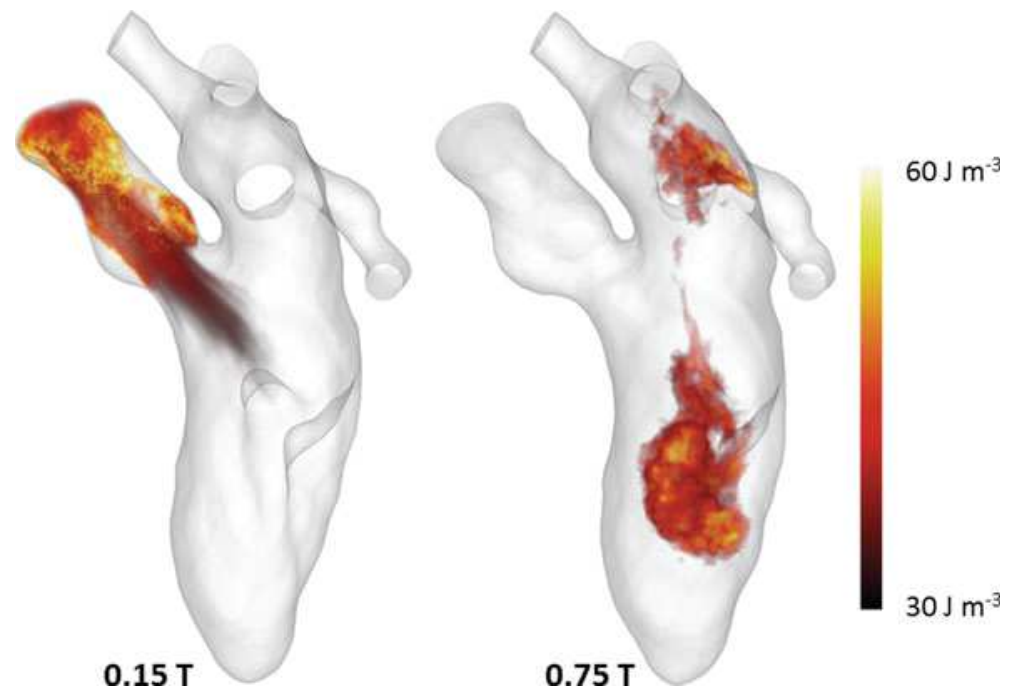

Fig. $93 \mathrm{D}$ volume rendering of the FKE in the left heart during systole (left, $0.15 \mathrm{~T}$ ) and diastasis (right, $0.75 \mathrm{~T}$ ). The opacity is set to $0 \%$ for FKE values below $30 \mathrm{~J} / \mathrm{m}^{3}$. The mitral and aortic valves are not displayed for more clarity but were accounted for in the simulation

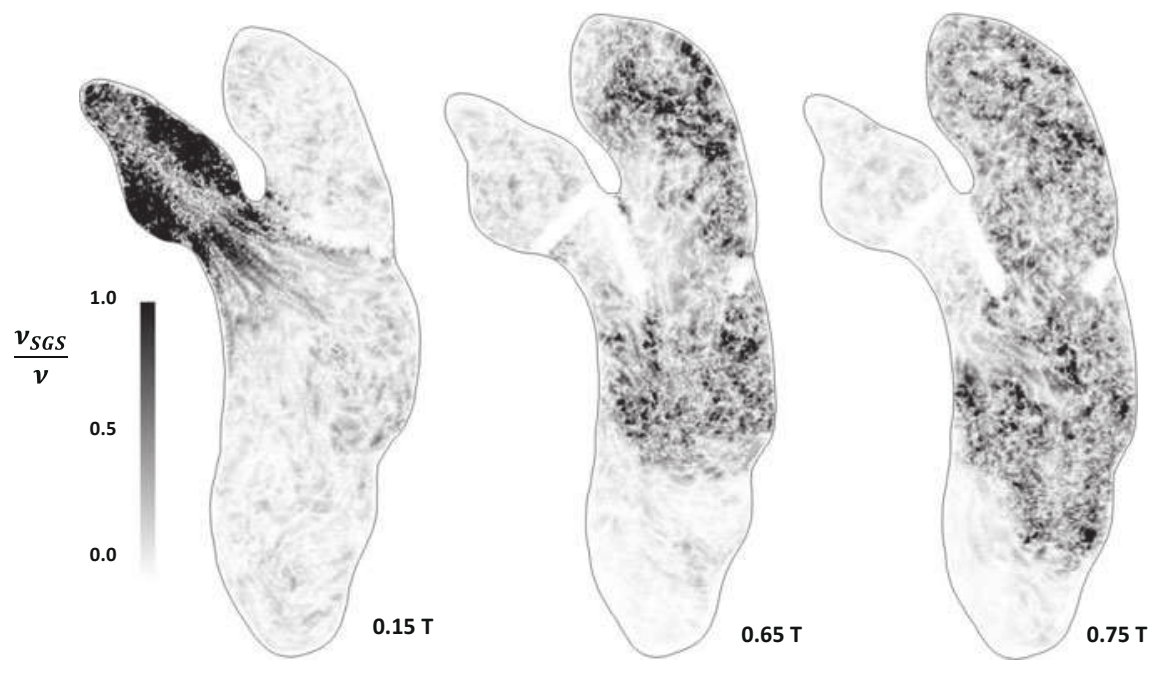

Fig. 10 Instantaneous fields of the SGS to fluid viscosity ratio. As expected the SGS viscosity tends to zero in the left ventricle during systole. The $v_{S G S} / \nu$ ratio reaches approx. 0.2 in average in the LV during the most disturbed phase of the heart cycle. Maximum values are close to 5

almost no dissipation in regions where the flow is laminar. This is one of the features of the $\sigma$-model that makes it suitable for computing cardiovascular flows. For this particular simulation, an estimation of the Pope criterion [50] can be computed 
as $k_{s g s} / k \approx 3 C / 2(\Delta / \pi L)^{2 / 3}$ [49] where $C=1.5, \Delta=0.55 \mathrm{~mm}$ and $L=0.04 \mathrm{~m}$ is the characteristic length of the largest structures. For the present LES, this leads to $k_{s g s} / k \approx 0.06$ which remains three times below the $15-20 \%$ threshold usually used to evaluate if a LES is sufficiently resolved [50].

\subsection{Discussion}

The present results question the common idea of laminar flow in the normal left heart. Imposing the flow to be laminar (as often done in CFD for the LV using commercial software) may be sufficient to retrieve main large-scale hemodynamic features, namely jets, recirculating cell, and ejection. However, transitional or turbulent regimes feature small-scale phenomena that cannot be retrieved under the laminar hypothesis. The typical Reynolds number value (a few thousand) would make the DNS of one single cardiac cycle extremely CPU/memory demanding, if not impossible. For example, Chnafa et al. [11] estimated that more than 1 billion nodes would be necessary to represent the Kolmogorov scale in their realistic human heart model. On the contrary, well-resolved large-eddy simulation allows retrieving the main characteristics of the flow and its transient properties at a lower cost. This allows simulating several cycles (here 35) at a tractable numerical cost, thus studying more complex flow features compared to what is usually done based on RANS simulations, including cycle-to-cycle variations.

\section{Cardiac Valves}

The aortic heart valve separates the left ventricle from the aorta. It is composed of three thin deformable leaflets that open and close passively during the cardiac cycle, preventing blood from flowing back into the left ventricle, and thus ensuring an unidirectional flow through the cardiovascular system. Aortic valves often degenerate and lead to either insufficiency or stenosis, which can cause the death of the patient if not treated. Usually, medication is not a sufficient treatment, and the aortic valve needs to be replaced by a prosthesis. One of the basic engineering concern for artificial aortic valve design is the hemodynamics. Indeed, the ideal aortic valve design should minimize production of turbulence which is notably known to have effect on thrombus formation (blood coagulation) [51, 52]. In Sect. 4, the aortic valve was represented thanks to a simple model fed by morphological data gained from medical imaging [11, 41, 43]. A more realistic modelling based on a Fluid-Structure Interaction (FSI) formulation is described in this section where an experimental configuration relevant to an artificial aortic valve [53] is also considered for validation. 


\section{Numerical Method}

The FSI model relies on the immersed thick boundary method (ITBM) [54] which was adapted from the original immersed boundary method (IBM) introduced by Peskin [55] to deal with 3D membranes. Two independent meshes are considered to discretize the solid valve and the fluid. The valve leaflets are represented by a moving Lagrangian mesh while the fluid is discretized by a fixed Eulerian unstructured mesh. The different steps of the ITBM are the following:

1. Knowing the displacement $\overrightarrow{U_{m}}$ of each Lagrangian node, the mechanical force $\overrightarrow{F_{m}}$ resulting from the membrane deformation is calculated,

2. The mechanical force $\overrightarrow{F_{m}}$ is regularized on the fluid mesh, giving rise to the volumetric force $\vec{f}_{j}$ on each fluid node,

3. The Navier-Stokes equations (forced by the regularized mechanical forces) are solved on the fluid mesh, yielding the velocity of the fluid $\overrightarrow{v_{j}}$ on each fluid node,

4. The velocity of the membrane $\overrightarrow{V_{m}}$ at each Lagrangian node is interpolated from the $\overrightarrow{v_{j}}$ field, enabling to deduce the new position $\overrightarrow{X_{m}}$ from the position at the previous timestep

$$
\overrightarrow{X_{m}}=\overrightarrow{X_{m}} \text { previous }+\Delta t \overrightarrow{V_{m}}
$$

The displacement of each Lagrangian node is then updated thanks to $\overrightarrow{U_{m}}=\overrightarrow{X_{m}}-$ $\overrightarrow{X_{m}^{0}}$, where $\overrightarrow{X_{m}^{0}}$ stands for the initial stress-free position (also referred to as the reference position).

The regularization and interpolation of steps 2 and 4 require dealing with Dirac functions that must be properly regularized on the Eulerian fluid mesh [56]. To this respect, the Reproducing Kernel Particle Method [57] is used to deal with unstructured meshes. The computation of the mechanical force $\overrightarrow{F_{m}}$ (step 1 ) is performed by the LMGC90 solid mechanics solver [58], using the classical finite-element method. In the present work, a quasi-incompressible Neo-Hookean material is used to model the valve, defined by its strain energy function:

$$
W=\frac{G}{2}\left(\overline{I_{1}}-3\right)+\frac{K}{2}(\ln J)^{2},
$$

where $G$ and $K$ are the shear and bulk modulus, respectively. $\overline{I_{1}}$ is the first invariant of the isochoric right Cauchy-Green deformation tensor $\overline{\mathbf{C}}$, and $J$ is the Jacobian of the transformation. 


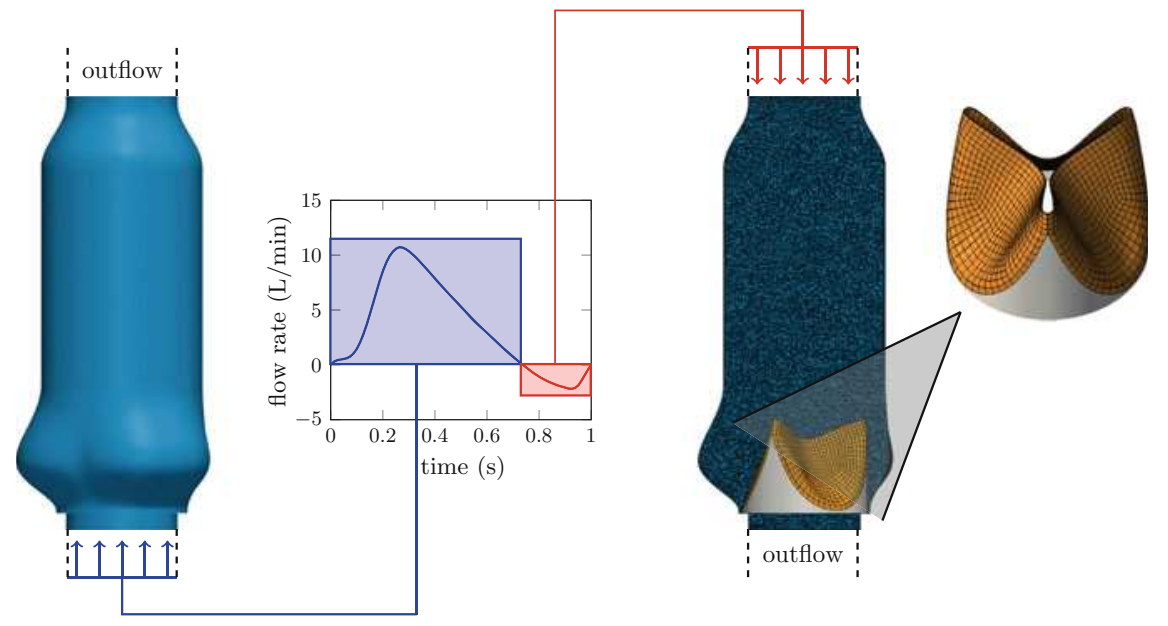

Fig. 11 Details of the computational setup. The flow is generated using switching inlet and outlet conditions, imposed far from the zone of interest. The assumption of a flat velocity profile is made to impose the inlet/outlet flow rate signal

\section{Computational Setup}

The computational setup was designed in such a way to represent the experimental test rig studied at the Helmholtz Institute of Aachen [53]. The aortic geometry includes the three sinuses of Valsalva after the calculations by Reul et al. [59] (see Fig. 11). The valve is built on a tri-commissure frame positioned within the aortic geometry, and is designed in an almost closed position (see Fig. 11). Both the arterial wall and the tri-commissure frame are defined as rigid bodies whose borders are non-slip wall boundary conditions of the fluid domain. As shown in Fig. 11, a flow rate signal is imposed as a periodic inlet condition either upstream or downstream of the valve, depending if the signal is positive or negative. The whole fluid geometry is represented by approx. 3.3 million tetrahedral elements, and the zone of interest (displayed in Fig. 11) is meshed with a uniform mesh resolution of $0.5 \mathrm{~mm}$. The valve mesh is composed of approx. 1500 quadratic hexahedral elements, with an effective resolution of $0.5 \mathrm{~mm}$.

The characteristics of the flow and the valve are relevant of physiological data and are summarized in Table 1. In view of the Reynolds $\left(R_{e}\right)$ and Womersley $\left(W_{0}\right)$ numbers, the viscosity of the Newtonian blood analogue fluid is set in the high-shear limit values [60].

\section{Results and Discussion}

Figure 12 shows the streamwise velocity field downstream of the valve. Four characteristic instants of the cardiac cycle are shown: $t=0.20 \mathrm{~s}$, just before the flow rate reaches its maximal value, referred to as Early Systole (ES); $t=0.26 \mathrm{~s}$, when the flow rate is maximal, referred to as Peak Systole (PS); $t=0.35 \mathrm{~s}$, just after the flow 
F. Nicoud et al.

Table 1 Characteristics of the flow and the valve set to reproduce physiological flow conditions

\begin{tabular}{l|l}
\hline Flow parameters & \\
\hline Density & $\rho=1100 \mathrm{~kg} / \mathrm{m}^{3}$ \\
\hline Dynamic viscosity & $\mu=3.6 \times 10^{-3} \mathrm{~Pa} \mathrm{~s}$ \\
\hline Heart rate & $n_{\text {bpm }}=60$ \\
\hline Mean cardiac output & $Q_{\text {mean }}=3.48 \mathrm{~L} / \mathrm{min}$ \\
\hline Reynolds number & $R_{e}=1388$ \\
\hline Womersley number & $W_{0}=17$ \\
\hline Valve parameters & \\
\hline Density & $\rho_{s}=1000 \mathrm{~kg} / \mathrm{m}^{3}$ \\
\hline Shear modulus & $G=2.4 \mathrm{MPa}$ \\
\hline Bulk modulus & $K=1.6 \mathrm{MPa}$ \\
\hline Thickness of the valve & $e=0.15 \mathrm{~mm}$ \\
\hline Radius of the valve & $R=12.5 \mathrm{~mm}$ \\
\hline
\end{tabular}

rate reaches its maximal value and begins to decrease, referred to as Mid-Systole (MS); $t=0.55 \mathrm{~s}$, when the flow rate is decreasing, referred to as Late Systole (LS). The main feature of the flow is the propagation of a jet emerging from the valve at ES (see label a), propagating between ES and MS (see labels b and c), and dissipating at LS. This jet is most probably hydro-dynamically unstable, promoting transition to turbulence downstream of the valve.

In order to analyse the structure of this jet and highlight the cycle-to-cycle variations of the flow, two velocity profiles are extracted over 10 distinct cycles (displayed in Fig. 12): profile 1 (P1), located immediately behind the valve; profile 2 (P2), located further downstream of the valve. Cycle-to-cycle variations are mainly observed on P2, suggesting that the jet emerging from the valve is mostly laminar and that it becomes turbulent when propagating further downstream. Regarding P2 at PS, MS and LS, it is seen that cycle-to-cycle variations increase as the inlet flow rate decreases, which is consistent with the observations made by Chnafa et al. [11, 41] in the case of whole heart geometries with simple (without FSI) valve model. This feature should be related to the general trend of all decelerated flows to destabilize. Few cycle-to-cycle variations are nonetheless observed on P1 at ES; these fluctuations are in fact induced by variations in the valve opening process and not related to any turbulent motion. This is illustrated in Fig. 13 which displays some typical shapes of the aortic valve. All these views correspond to the same three instants (the third one corresponding to ES) but were extracted at four different cycles. If the overall opening process is always the same (buckling of the leaflets, either successive or simultaneous), very large fluctuations are also present from one cycle to the other. These variations most probably result from two main ingredients: the sensitivity of the buckling phenomenon to the details of the load on the first hand, the existence of a chaotic pressure load induced by the downstream turbulent fluctuations on the other hand. 
Large-Eddy Simulation of Turbulence in Cardiovascular Flows

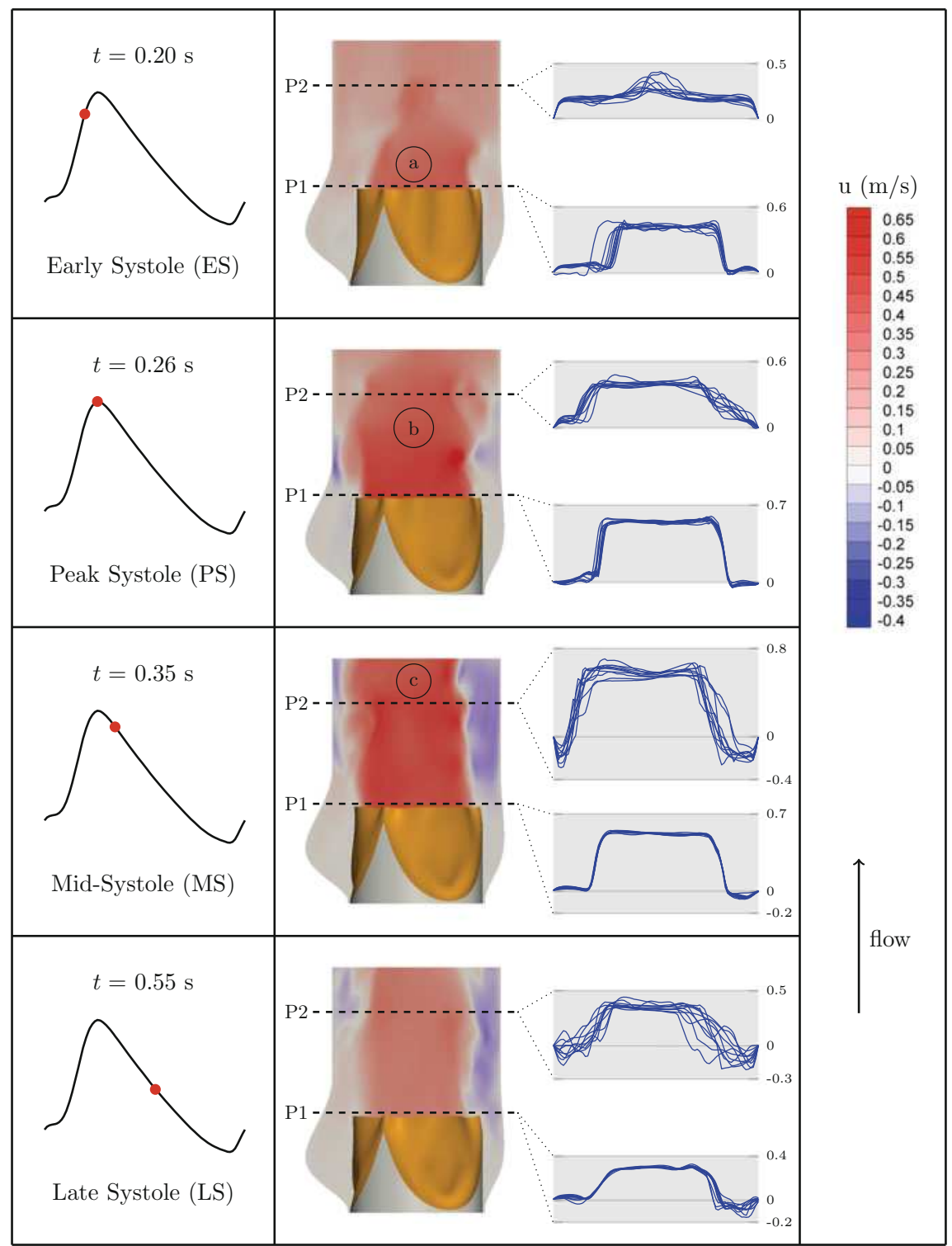

Fig. 12 Streamwise velocity field downstream of the valve. Two velocity profiles (P1 and P2) are extracted over 10 distinct cycles. Four different instants of the cardiac cycle are depicted (ES, PS, MS and LS) 


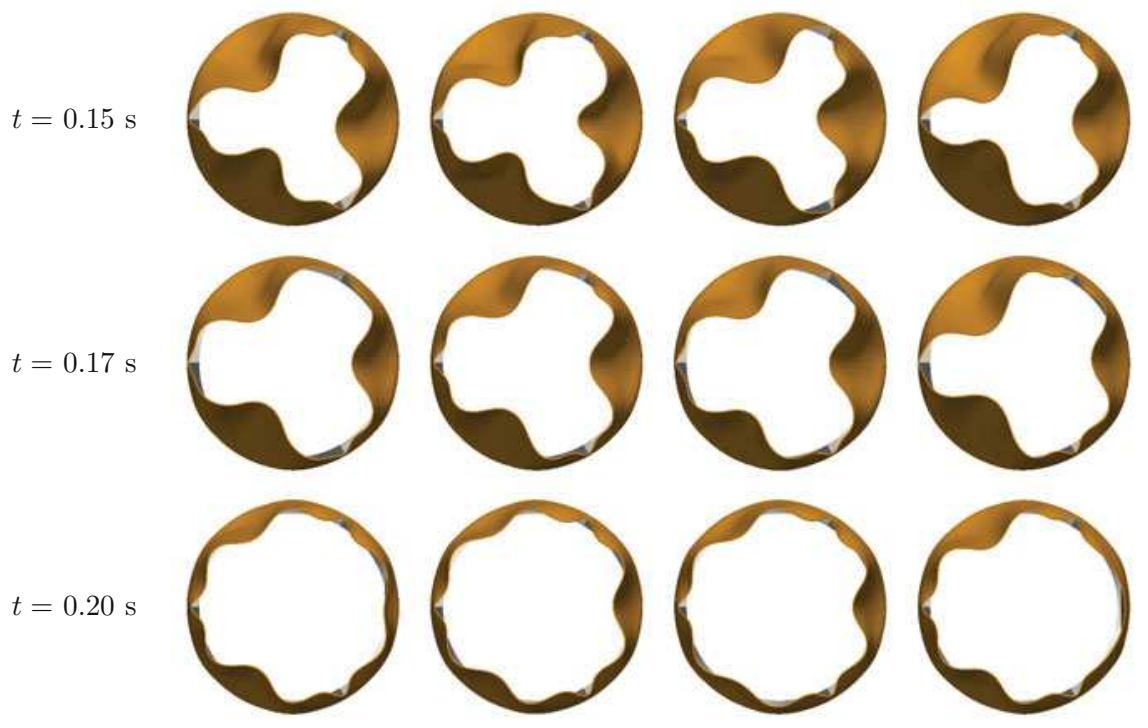

Fig. 13 Typical shapes of the aortic valve during the opening phase. The last instant $(t=0.20 \mathrm{~s})$ corresponds to ES (see Fig. 12). Each column corresponds to a specific cycle

\section{Conclusion}

Given the flow regimes encountered in macro-circulation, turbulence is most probably present in many practical configurations, either physiological or within biomedical devices. Large-Eddy Simulation has the potential to properly represent the effects of turbulence provided that it is used with appropriate numerics, subgrid scale model and boundary conditions. The numerical solver YALES2BIO (http://www. math.univ-montp2.fr/ yales2bio/) gathers all the required properties and was successfully used in three different situations including the intracardiac flow and the interaction with a realistic aortic valve. Transitional flows are even more demanding and should be addressed very carefully since they may be extremely sensitive to the details of the simulation if no perturbation is introduced in the upstream condition. This is illustrated by considering the simplified medical device proposed by FDA as a validation test case.

Acknowledgements The authors thank ANR and BPI for their supports through the Laboratory of Excellence NUMEV (ANR-10-LABX-20), the FORCE (ANR-11-JS09-0011) and the DAT@DIAG (ISI-I1112018W) project. Dr S. Sonntag and PhD student D. Pott from the Helmholtz Institute of Aachen are gratefully acknowledged for providing the details of their experimental test rig about the aortic valve dynamics. CC also thanks CNRS for funding his thesis. Dr. V. Moureau is gratefully acknowledged for giving access to the YALES2 solver. This work was performed using HPC resources from GENCI-CINES (Grants 2014-, 2015- and 2016-c2015037194) and the HPC@LR Center. 
Large-Eddy Simulation of Turbulence in Cardiovascular Flows

\section{References}

1. S. Varghese, S. Frankel, P. Fischer, Direct numerical simulation of stenotic flows. Part 2. Pulsatile flow. J. Fluid Mech. 582, 281 (2007)

2. A. Les, S. Shadden, C. Figueroa, J. Park, M. Tedesco, R. Herfkens, R. Dalman, C. Taylor, Quantification of hemodynamics in abdominal aortic aneurysms during rest and exercise using magnetic resonance imaging and computational fluid dynamics. Ann. Biomed. Eng. 38(4), 1288-1313 (2010)

3. K. Valen-Sendstad, M. Piccinelli, D. Steinman, High-resolution computational fluid dynamics detects flow instabilities in the carotid siphon: implications for aneurysm initiation and rupture? J. Biomech. 47(12), 3210-3216 (2014)

4. J. Mikhal, B. Geurts, Immersed boundary method for pulsatile transitional flow in realistic cerebral aneurysms. Comput. Fluids 91, 144-163 (2014)

5. F. Domenichini, G. Querzoli, A. Cenedese, G. Pedrizzetti, Combined experimental and numerical analysis of the flow structure into the left ventricle. J. Biomech. 40, 1988-1994 (2007)

6. P. Dyverfeldt, J. Kvitting, C. Carlhäll, G. Boano, A. Sigfridsson, U. Hermansson, A. Bolger, J. Enqwall, T. Ebbers, Hemodynamic aspects of mitral regurgitation assessed by generalized phase-contrast MRI. J. Magn. Reson. Imaging 33, 582-588 (2011)

7. J. Zajac, J. Eriksson, P. Dyverfeldt, A. Bolger, T. Ebbers, C. Carlhäll, Turbulent kinetic energy in normal and myopathic left ventricles. J. Magn. Reson. Imaging 41(4), 1021-1029 (2015)

8. U. Frisch, Turbulence: The Legacy of A.N. Kolmogorov (Cambridge University Press, 1996)

9. P. Spalart, Progress in aerospace sciences philosophies and fallacies in turbulence modeling. Prog. Aerosp. Sci. 1-15 (2015)

10. P. Hariharan, M. Giarra, V. Reddy, S. Day, K. Manning, S. Deutsch, S. Stewart, M. Myers, M. Berman, G. Burgreen, E. Paterson, R. Malinauskas, Multilaboratory particle image velocimetry analysis of the FDA benchmark nozzle model to support validation of computational fluid dynamics simulations. J. Biomech. Eng. 133(4), 041002 (2011)

11. C. Chnafa, S. Mendez, F. Nicoud, Image-based large-eddy simulation in a realistic left heart. Comput. Fluids 94, 173-187 (2014)

12. P. Sagaut, Large Eddy Simulation for Incompressible Flows. An Introduction (Springer, Berlin, 2001)

13. R. Vichnevetsky, J. Bowles, Fourier Analysis of Numerical Approximations of Hyperbolic Equations, Siam - stu edn. (1982)

14. Y. Morinishi, T. Lund, O. Vasilyev, P. Moin, Fully conservative higher order finite difference schemes for incompressible flow. J. Comput. Phys. 143(1), 90-124 (1998)

15. R. Mittal, P. Moin, Suitability of upwind-biased finite differance schemes for large eddy simulation of turbulent flows. AIAA J. 35(8), 1415-1417 (1997)

16. N. Park, J. Yoo, H. Choi, Discretization errors in large eddy simulation: on the suitability of centered and upwind-biased compact difference schemes. J. Comput. Phys. 198(2), 580-616 (2004)

17. A. Chorin, Numerical solution of the Navier-Stokes equations. Math. Comput. 22, 745-762 (1968)

18. J. Williamson, Low-storage Runge-Kutta schemes. J. Comput. Phys. 35(1), 48-56 (1980)

19. V. Moureau, P. Domingo, L. Vervisch, Design of a massively parallel CFD code for complex geometries. Comptes Rendus Mécanique 339(2-3), 141-148 (2011)

20. M. Malandain, N. Maheu, V. Moureau, Optimization of the deflated Conjugate Gradient algorithm for the solving of elliptic equations on massively parallel machines. J. Comput. Phys. 238, 32-47 (2013)

21. F. Nicoud, H. Baya Toda, O. Cabrit, S. Bose, J. Lee, Using singular values to build a subgridscale model for large eddy simulations. Phys. Fluids 23(8), 085106 (2011)

22. H. Baya Toda, O. Cabrit, K. Truffin, G. Bruneaux, F. Nicoud, Assessment of subgrid-scale models with a large-eddy simulation-dedicated experimental database: the pulsatile impinging jet in turbulent cross-flow. Phys. Fluids 26(7), 075108 (2014) 
23. M. Germano, U. Piomelli, P. Moin, W. Cabot, A dynamic subgrid-scale eddy viscosity model. Phys. Fluids A 3, 1760-1766 (1991)

24. S. Stewart, E. Paterson, G. Burgreen, P. Hariharan, M. Giarra, V. Reddy, S. Day, K. Manning, S. Deutsch, M. Berman, M. Myers, R. Malinauskas, M. Berman, R. Malinauskas, Assessment of CFD performance in simulations of an idealized medical device: results of FDA's first computational interlaboratory study. Cardiovasc. Eng. Technol. 3(2), 139-160 (2012)

25. A. Marsden, Y. Bazilevs, C. Long, M. Behr, Recent advances in computational methodology for simulation of mechanical circulatory assist devices. WIREs Syst. BiolMed. 6, 169-188 (2014)

26. S. Mendez, V. Zmijanovic, E. Gibaud, J. Siguenza, F. Nicoud, Assessing macroscopic models for hemolysis from fully resolved simulations, in 4th International Conference on Computational and Mathematical Biomedical Engineering, CMBE2015 Proceedings, ed. by P. Nithiarasu, E. Budyn (ENS Cachan, France, 2015), pp. 575-578

27. T. Passerini, A. Quaini, U. Villa, A. Veneziani, S. Canic, Validation of an open source framework for the simulation of blood flow in rigid and deformable vessels. Int. J. Numer. Methods Biomed. Eng. 29(11), 1192-1213 (2013)

28. Y. Delorme, K. Anupindi, S. Frankel, Large eddy simulation of FDA's idealized medical device. Cardiovasc. Eng. Technol. 4(4), 392-407 (2013)

29. S. Bhushan, D. Walters, G. Burgreen, Laminar, turbulent, and transitional simulations in benchmark cases with cardiovascular device features. Cardiovasc. Eng. Technol. 4(4), 408-426 (2013)

30. G. Janiga, Large eddy simulation of the FDA benchmark nozzle for a Reynolds number of 6500. Comput. Biol. Med. 47(April), 113-119 (2014)

31. F. Sotiropoulos, Computational fluid dynamics for medical device design and evaluation: are we there yet? Cardiovasc. Eng. Technol. 3(2), 137-138 (2012)

32. V. Zmijanovic, S. Mendez, V. Moureau, F. Nicoud, About the numerical robustness of biomedical benchmark cases: Interlaboratory FDA's idealized medical device. Int. J. Numer. Methods Biomed. Eng. (2016). doi:10.1002/cnm.2789

33. X. Wu, P. Moin, R. Adrian, J. Baltzer, Osborne Reynolds pipe flow: direct simulation from laminar through gradual transition to fully developed turbulence. Proc. Natl. Acad. Sci. 112(26), 7920-7924 (2015)

34. K. Avila, D. Moxey, A. de Lozar, M. Avila, D. Barkley, B. Hof, The onset of turbulence in pipe flow. Science 333, 192-196 (2011)

35. A. Kheradvar, M. Gharib, On mitral valve dynamics and its connection to early diastolic flow. Ann. Biomed. Eng. 37(1) (2009)

36. G. Pedrizzetti, F. Domenichini, G. Tonti, On the left ventricular vortex reversal after mitral valve replacement. Ann. Biomed. Eng. 38(3), 769-773 (2010)

37. P. Davies, A. Remuzzi, E. Gordon, C. Dewey, M. Gimbrone, Turbulent fluid shear stress induces vascular endothelial cell turnover in vitro. Proc. Natl. Acad. Sci. 83(7), 2114-2117 (1986)

38. S. Olesen, D. Clapham, P. Davies, Haemodynamic shear stress activates a K+ current in vascular endothelial cells. Nature 331(6152), 168-170 (1988)

39. A. Pasipoularides, Mechanotransduction mechanisms for intraventricular diastolic vortex forces and myocardial deformations: Part 1. J. Cardiovasc. Transl. Res. 8(1), 76-87 (2015)

40. F. Domenichini, G. Pedrizzetti, B. Baccani, Three-dimensional filling flow into a model left ventricle. J. Fluid Mech. 539, 179-198 (2005)

41. C. Chnafa, S. Mendez, F. Nicoud, Image-based simulations show important flow fluctuations in a normal left ventricle: what could be the implications? Ann. Biomed. Eng. (2016). doi:10. 1007/s10439-016-1614-6

42. T. Le, F. Sotiropoulos, On the three-dimensional vortical structure of early diastolic flow in a patient-specific left ventricle. Eur. J. Mech. B/Fluids 35, 20-24 (2012)

43. C. Chnafa, S. Mendez, R. Moreno, F. Nicoud, Using image-based CFD to investigate the intracardiac turbulence, in Modeling the Heart and the Circulatory System, ed. by A. Quarteroni (Springer International Publishing, New York, 2015), pp. 97-117 
Large-Eddy Simulation of Turbulence in Cardiovascular Flows

44. P. Kilner, G. Yang, J. Wilkes, R. Mohiaddin, D. Firmin, M. Yacoub, Asymmetric redirection of flow through the heart. Nature 404(6779), 759-761 (2000)

45. V. Mihalef, R. Ionasec, P. Sharma, B. Georgescu, I. Voigt, M. Suehling, D. Comaniciu, Patient-specific modelling of whole heart anatomy, dynamics and haemodynamics from fourdimensional cardiac CT images. Interface Focus 1(3), 286-296 (2011)

46. A. Falahatpisheh, A. Kheradvar, High-speed particle image velocimetry to assess cardiac fluid dynamics in vitro: From performance to validation. Eur. J. Mech. B/Fluids 35, 2-8 (2012)

47. G. Querzoli, S. Fortini, A. Cenedese, Effect of the prosthetic mitral valve on vortex dynamics and turbulence of the left ventricular flow. Phys. Fluids 22, 1-10 (2010)

48. I. Celik, Z. Cehreli, I. Yavuz, Index of resolution quality for large eddy simulations. J. Fluid Eng. 127(5), 949-958 (2005)

49. S. Pope, Turbulent Flows (Cambridge University Press, 2000)

50. S. Pope, Ten questions concerning the large-eddy simulation of turbulent flows. New J. Phys.6 (2004)

51. P. Stein, H. Sabbah, Measured turbulence and its effect on thrombus formation. Circ. Res. 35, 608-614 (1974)

52. A. Yoganathan, Z. He, S. Jones, Fluid mechanics of heart valves. Annu. Rev. Biomed. Eng. 6, 331-362 (2004)

53. D. Pott, J. Sigüenza, S. Sonntag, U. Steinseifer, S. Mendez, F. Nicoud, Dynamics of artificial aortic valves: a combined experimental and numerical study, in 42th ESAO meeting, Leuven (2015)

54. J. Sigüienza, S. Mendez, D. Ambard, F. Dubois, F. Jourdan, R. Mozul, F. Nicoud, Validation of an immersed thick boundary method for simulating fluid-structure interactions of deformable membranes. J. Comput. Phys. 322, 723-746 (2016). doi:10.1016/j.jcp.2016.06.041, http://dx. doi.org/10.1016/j.jcp.2016.06.041

55. C. Peskin, The immersed boundary method. Acta Numerica 11, 479-517 (2002)

56. S. Mendez, E. Gibaud, F. Nicoud, An unstructured solver for simulations of deformable particles in flows at arbitrary Reynolds numbers. J. Computat. Phys. 256, 465-483 (2014)

57. A. Pinelli, I. Naqavi, U. Piomelli, J. Favier, Immersed-boundary methods for general finitedifference and finite-volume Navier-Stokes solvers. J. Comput. Phys. 229, 9073-9091 (2010)

58. F. Radjai, F. Dubois, Discrete Numerical Modeling of Granular Materials (Wiley-ISTE, 2011)

59. H. Reul, A. Vahlbruch, M. Giersiepen, T. Schmitz-Rode, V. Hirtz, S. Effert, The geometry of the aortic root in health, at valve disease and after valve replacement. J. Biomech. 23(2), 181-191 (1990)

60. A. Robertson, A. Sequeira, R. Owens, Rheological models for blood, in Cardiovascular Mathematics. Modeling and Simulation of the Circulatory System (Springer, 2009), pp. 211-241 